Canadian

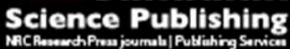

Applied Physiology, Nutrition, and Metabolism Physiologie appliquée, nutrition et métabolisme

\title{
Associations between physical activity, screen time, and fitness among 6- to 10-year old children living in Edmonton, Canada
}

\begin{tabular}{|r|l|}
\hline Journal: & Applied Physiology, Nutrition, and Metabolism \\
\hline Manuscript ID & apnm-2016-0419.R2 \\
\hline Manuscript Type: & Article \\
\hline Date Submitted by the Author: & 09-Dec-2016 \\
\hline Complete List of Authors: & $\begin{array}{l}\text { Potter, Morgan; University of Alberta, Faculty of Physical Education and } \\
\text { Recreation } \\
\text { Spence, John; University of Alberta, Faculty of Physical Education and } \\
\text { Recreation } \\
\text { Boulé, Normand; University of Alberta, } \\
\text { Stearns, Jodie; University of Alberta, Faculty of Physical Education and } \\
\text { Recreation } \\
\text { Carson, Valerie; University of Alberta, Faculty of Physical Education and } \\
\text { Recreation }\end{array}$ \\
\hline Keyword: & fitness, screen time, physical activity < exercise, children, Canada \\
\hline \multicolumn{2}{|c}{} \\
\hline
\end{tabular}


Associations between physical activity, screen time, and fitness among 6- to 10-year old children living in Edmonton, Canada

Morgan Potter, John C. Spence, Normand G. Boulé, Jodie A. Stearns, Valerie Carson

Corresponding Author:

Valerie Carson, $\mathrm{PhD}$

1-151 Van Vliet Complex

University of Alberta

Edmonton, AB. T6G 2H9

Phone: (780) 492-1004

Fax: (780) 492-1008

E-mail: vlcarson@ualberta.ca

M. Potter, J.C. Spence, N.G. Boulé, J.A. Stearns, and V. Carson. Faculty of Physical

Education and Recreation, University of Alberta, Edmonton, AB, T6G 2H9, Canada.

Email contact:

M. Potter (mrwagner@ualberta.ca); J.C. Spence (jc.spence@ualberta.ca); N.G. Boulé

(nboule@ualberta.ca); J. A. Stearns (jodie.stearns@ualberta.ca); V. Carson

(vlcarson@ualberta.ca) 


\begin{abstract}
The objectives of this study were to describe fitness levels, examine associations between physical activity (PA), screen time (ST), and fitness, and examine sex moderating effects in a sample of children. Participants were 649 children ( $7.8 \pm 0.6$ years; $52.4 \%$ female) from Edmonton, Canada. Hours/week of PA and ST were parent-reported. PA was also objectively measured with pedometers and expressed in increments of 1000 steps/day. Fitness components (i.e., vertical jump, sit-and-reach, waist circumference, grip strength, predicted maximal oxygen consumption $\left(\mathrm{VO}_{2}\right.$ max $)$, push-ups, partial curl-ups, overall fitness) were measured according to the Canadian PA, Fitness and Lifestyle Approach protocols and expressed as z-scores or low/high fitness. Positive associations were observed between PA and overall fitness for both the complete (Subjective: $\beta=0.009,95 \% \mathrm{CI}: 0.001,0.017)$ and partial $(\geq 3$ available fitness measures; Subjective: $\beta=0.006,95 \%$ CI: 0.000, 0.011 ; Objective: $\beta=0.025,95 \% \mathrm{CI}$ : $0.007,0.042$ ) fitness scores. Subjective $(\beta=0.011,95 \%$ CI: $0.000,0.022)$ and objective $(\beta=0.043,95 \%$ CI: 0.008 , 0.078) PA were positively associated with vertical jump. Children with higher objective PA were more likely to be in the high push-ups group (OR=1.156, 95\%CI: $1.054,1.267)$. PA was positively associated with predicted $\mathrm{VO}_{2} \max$ (Subjective: $\beta=0.040,95 \% \mathrm{CI}$ : 0.018, 0.063; Objective: $\beta=0.084,95 \%$ CI: $0.012,0.157)$ and grip strength (Subjective: $\beta=0.025,95 \%$ CI: 0.011 , $0.040)$ in boys only. ST was negatively associated with grip strength $(\beta=-0.016,95 \% \mathrm{CI}:-0.028,-$ 0.004) in boys. PA was associated with several components of fitness, especially in boys. However, few associations were observed between ST and fitness. Promoting regular PA in young children may address declining fitness levels.
\end{abstract}

Keywords: fitness, screen time, physical activity, children, Canada, cross-sectional 


\section{Introduction}

Low health-related fitness in children, including cardiovascular, musculoskeletal, flexibility, and body composition, is associated with risk factors for chronic diseases (Grontved et al. 2013;

Ortega et al. 2008; Garcia-Artero et al. 2007). Conversely, higher levels of fitness are associated with reduced blood pressure, less abdominal obesity, improved bone mineral density, and reduced depression, anxiety, and stress (Ortega et al. 2008; Tremblay et al. 2010; Janssen and Leblanc 2010). Despite the clear health benefits, children's fitness levels have decreased significantly since the 1980's in Canada and internationally (Tremblay et al. 2010; Tomkinson et al. 2003). Consequently, it is important to understand the factors that predict fitness, especially in childhood when health behaviours are established, to reverse these trends.

Some research in children suggests that fitness is determined in part by non-modifiable factors, such as genetics (Guth and Ross 2013; Chiu et al. 2011) and low birth weight (van Deutekom et al. 2015). Physical fitness may also be influenced by modifiable factors such as, physical activity (PA) and sedentary behaviour (Santos et al. 2015). More specifically, it is possible that increasing energy output through PA is associated with notable changes in physiological variables, such as blood lipids and blood pressure, which can in turn impact physical fitness levels (Blair et al. 2001). It is likely that the reverse could be present with increased screen time (ST), as increasing sedentary behaviours reduces energy output.

The PA and ST of children and youth has been a key public health focus over the past two decades (Tremblay et al. 2010; ParticipACTION 2016). However, research indicates that children's PA remains extremely low (Colley et al. 2011; Tremblay et al. 2010; Shields 2006) and many interventions have been developed and implemented to target this behaviour in multiple settings (Showell et al. 2013; Harris et al. 2009), but with limited success (Metcalf et al. 2012). Currently only $14 \%$ of Canadian children aged 5 to 11 years are meeting the 
recommended PA guidelines of 60 minutes of moderate-to-vigorous PA each day (ParticipACTION 2016). Similar to PA, only 24\% of children aged 5 to 11 years meet the ST recommendations of less than two hours each day (ParticipACTION 2016) within the sedentary behaviour guidelines for Canadian children (CSEP, 2014b). Current low levels of PA and high levels of ST may partially explain the declining fitness levels.

To date, fitness levels and factors affecting fitness in children less than 10-years of age, such as PA and ST, have been relatively unexplored (Smith et al. 2014). Furthermore, the role of sex in these relationships is unclear. Children naturally display an increase in strength until puberty; however, girls' fitness tends to plateau and boy's fitness tends to increase after puberty (Beunen and Thomis 2000; Gallahue and Ozman 2006). Since these differences exist in adolescence, it might be worthwhile to consider whether sex impacts the associations between PA or ST and fitness in children. Therefore, the objectives of this paper are to: (1) describe fitness levels in a sample of 6- to 10-year-olds from Edmonton, Canada; (2) examine the associations between PA, ST, and fitness; and (3) examine if sex moderates the associations between PA, ST, and fitness.

\section{Materials and methods}

\section{Participants}

Participants for this cross-sectional study were a part of the Spatial Health Assessment of Physical Environments (SHAPE) baseline study and the SHAPEs of Things to Come longitudinal follow-up study. During the baseline phase (2005-2007), children aged 4- to 6-years and their parents were recruited from Capital Health Centres when the children received their preschool immunizations. Though preschool immunization is not mandatory in the province of Alberta, prior research indicated that $74 \%$ of children in the Edmonton region were given the 
immunization for Diphtheria, Tetanus, Pertussis and Polio (DTap-IPV) before entering grade one in 2004 (Edwards et al. 2008). The percentage of early years children immunized for DTap-IPV in 2014 stayed relatively consistent at 76\% (Government of Alberta 2015). This suggests that most children accessed these health centres before entering grade one, making the centres an appropriate place to reach a large proportion of children from the Edmonton health region.

A total of 2,114 children participated in the baseline study. Of these, 1,337 (63\%) agreed to be re-contacted for future research. Once contacted, 649 aged 6- to 10-years participated in the follow-up data collection. This study is based on the follow-up data collection only, since fitness was only measured at that time. The University of Alberta's Ethics Board (HREB) approved this study and all parents provided written and informed consent.

\section{Procedures}

Children whose parents agreed to be contacted regarding follow-up research during the baseline data collection were contacted by telephone. If the parents remained interested in participating they were sent an information form, consent form, and a short questionnaire. All fitness testing was done at the University of Alberta campus. Once at the facility, the consent form and brief questionnaire were collected from the parent. The procedures were then explained to both the parent and child, and the child performed the physical fitness assessment. At the end of the session, children were provided a pedometer to take home. They were asked to wear the pedometer for four consecutive days, including three weekdays and one weekend day (i.e., Sunday to Wednesday or Wednesday to Saturday), and parents recorded the total number of steps at the end of each day in a logbook. Families received a small thank-you gift for participating (e.g. gift cards, pencils, stickers, flash cards) valued at approximately $\$ 20$ per family. 


\section{Measures}

\section{Exposure Variables}

Subjective PA: A modified version of the Children's Leisure Activities Study Survey (CLASS; Telford et al. 2004) was used to collect parent-reported PA of their child. Using a proxy-report measure in this age group reduces recall errors that may be present due to cognitive limitations (Sirard and Pate 2001). Specifically, they reported on nine PA's that the child usually participated in during the current season: swimming (lessons and for fun), soccer, ballet/dance, gymnastics, skating, hockey, bike riding, gym activities, and active play (including at a playground). A tenth space was provided for inclusion of a PA that was not listed in the first nine options. Parents recorded frequency (how many times) and duration (average minutes each time) for each activity done on a weekday (Monday - Friday) and weekend (Saturday-Sunday). Total hours per week of subjective PA were calculated by summing weekday and weekend minutes and dividing by 60 . PA values greater than 42 hours per week were defined as outliers and truncated to 42 hours $(n=6)$. Previous work has shown substantial to almost perfect 2 -week testretest reliability (62\%-94\% agreement) for the CLASS when estimating PA for children ages 5 to 6 years (Telford et al. 2004). Similar findings have been observed for 10 to 12 year olds (Telford et al. 2004).

Objective PA: Children's PA was also measured objectively using a StepsCount SC-T2 [Steps Count, Deep River, ON, CA] pedometer. Additional information on the use of this pedometer has been published elsewhere (Stearns et al. 2016). Parents and children were instructed that the child should put the pedometer on as soon as they awoke in the morning. Once the pedometer was in place and positioned above the right hip, participants were to walk 20 steps and check that the pedometer recorded between 19 and 21 steps. If this did not happen, 
participants were instructed to re-position the pedometer and try again. Pedometers were not to be worn while swimming or bathing. Participants removed the pedometer before going to bed and parents recorded the number of steps taken at the end of the day in the activity log. Participants with at least three days of complete pedometer data were included. Average steps per day were calculated by averaging across valid days. For regression analyses, steps per day were expressed in increments of 1000 steps/day (steps per day divided by 1000). Pedometers have shown acceptable reliability $(r=0.79)$ for measuring PA in elementary school children (mean age $=9.3$-years) when worn for at least three days (Ling and King 2015).

ST: Children's ST was assessed through parent-reported usual participation in three ST activities: TV/videos, play station/Nintendo/x-box/ game boy, and computer/internet/computer games. An additional space was provided for the inclusion of a ST activity that was not mentioned previously. Parents recorded the total amount of time their child participated in these activities in a typical week and a typical weekend. Average hours per week of ST were calculated by summing weekday and weekend day minutes and dividing by 60 . Values greater than 42 hours per week were considered outliers and were truncated to 42 hours of weekly ST $(n=6)$. This measure of ST has been included in a previously published study (Carson et al. 2010); however, data on psychometric properties are not available.

\section{Outcome Variables}

The Canadian Physical Activity, Fitness \& Lifestyle Approach (CPAFLA) manual and fitness testing protocol was used to assess physical fitness (CSEP 2003). At the time of data collection, this was the current protocol for the Canadian Society for Exercise Physiology (CSEP). At least one of the research assistants in every data collection session was recognized as a Certified Personal Trainer through programs offered by CSEP. The battery of fitness tests 
measured anthropometry, aerobic fitness, musculoskeletal fitness, and flexibility. A Physical Activity Readiness Questionnaire (PAR-Q; CSEP 2014a) was completed by parents prior to children participating in the physical fitness tests. Also, participants were pre-screened using a heart rate measure. Resting heart rate less than 100 beats per minute was used as the prescreening cut off. If heart rate exceeded 100 beats per minute, participants were instructed to wait seated for five minutes before being re-tested. Only participants who met the resting heart rate requirements on the first or second measures were allowed to proceed with the testing. All participants met the heart rate requirements and completed the tests in the order described below.

Anthropometric measure: Waist circumference was the anthropometric measure used. A non-stretch tape measure was used to assess waist girth to the nearest $0.1 \mathrm{~cm}$ at the top border of the iliac crest. Each participant's waist circumference was measured twice, and if the two measures differed by more than $0.5 \mathrm{~cm}$ a third measure was taken. The final waist circumference value consisted of an average of the two or three trials.

Musculoskeletal Fitness: Musculoskeletal fitness was measured using grip strength, pushups, partial curl-ups, and vertical jump. Grip strength was used to measure overall muscular strength using a dynamometer. The participants were asked to stand upright with their arms slightly raised to the sides and alternate squeezing the dynamometer in each hand twice, resting between each try. The output for each hand was recorded to the nearest 1.0 kilograms by the examiner. The highest outputs for each hand were summed.

Push-ups measured upper body muscular endurance. Boys completed push-ups pivoting from the toes and girls pivoted from the knees. Participants were instructed to lay prone on a mat with their hands underneath their shoulders and palms on the ground. Pivoting from either the toes or knees, participants completed push-ups until failure. The participants had to fully 
straighten their arms at the top of the push-up and lower their body until their chin was near the mat, without their stomach touching the mat. If any push-ups were done improperly, the examiner would verbally correct the participants form. If done incorrectly a second time the child was told to stop the test. Incorrect repetitions did not count. The number of correct completed push-ups before failure was recorded.

Partial curl-ups tested abdominal endurance. Participants were instructed to lay supine on a mat with their arms resting palms down beside their body with their knees bent at ninety degrees. Participants were to slide their hands forward on the mat 10 centimeters, as marked with tape, and move back to resting position. This was done to a set cadence of 50 beats per minute. The number of partial curls-ups done in one minute was recorded (maximum of 25 repetitions).

Vertical jump measured lower body power. To record standing reaching height, participants stood perpendicular to a wall with their feet flat on the floor and one arm extended up the wall. Next, the participant stood slightly away from the wall (with hands on the hips, elbow should almost be able to touch the wall). With no pre-jump, the participant was instructed to bend their knees, pause briefly, and jump as high as they could, and touching the wall at the peak jump height. The peak jump height was tested three times, with a rest period of 15 seconds between each attempt. The standing reaching height was subtracted from the best jump height and recorded as vertical jump height to the nearest $0.5 \mathrm{~cm}$.

Flexibility: Sit-and-reach measured hamstring and lower back flexibility. Before beginning this test, participants were instructed to stretch their lower back, hamstrings, and calves. A device was set up to measure forward reach and participants were instructed to take off their shoes, sit on the floor with legs fully extended, and press their feet against the base of the device. With arms fully extended, hands overlapped with palms down, participants gently 
reached as far forward as possible and paused for 2 seconds. This was done twice and the maximum reach between the two trials was recoded to the nearest $0.5 \mathrm{~cm}$.

Aerobic Fitness: Aerobic fitness was measured using the modified Canadian Aerobic Fitness Test (mCAFT). The mCAFT protocol involves stepping up and down two stairs to a set cadence. The cadence is played through an audio recording that increases in intensity as participant's progress through the stages. Boys started the step test at stage four and girls started at stage three. Participants progressed through the stages until they reached $85 \%$ of their agepredicted heart rate max (220-age; CSEP 2003). Post exercise heart rate was measured 2, 3, and 4 minutes after completion of the exercise. Predicted maximal aerobic power $\left(\mathrm{VO}_{2} \max \right)$ was calculated for participants who reached $85 \%$ of their age-predicted maximal heart rate using the Weller Equation (Weller et al. 1995). This equation is typically used to predict $\mathrm{VO}_{2}$ max in adults, but has been used previously with children (Tremblay et al. 2010).

Vertical jump, waist circumference, sit-and-reach, predicted $\mathrm{VO}_{2}$ max, and grip strength were expressed as sex-specific z-scores specific to this sample. The values for the push-up and partial curl-up test were not normally distributed and did not improve with transformation; therefore, participants were categorized into "high" and "low" fitness using the age and sex specific standards outlined for gold in the Canada Fitness Award Manual (1986) as a cut off. For example, a 10-year old boy would meet the gold standard if they completed at least 20 push-ups and 30 partial curl-ups; whereas, a 10-year old girl would meet the gold standard if they completed at least 19 push-ups and 28 partial curl-ups (Canada Fitness Award Manual 1986).

Overall fitness: Predicted $\mathrm{VO}_{2}$ max, grip strength, vertical jump, waist circumference, and sit-and-reach z-scores were used to create two total fitness scores. "Fitness-complete" reflects the mean of the five fitness $\mathrm{z}$-scores (predicted $\mathrm{VO}_{2}$ max, grip strength, vertical jump, 
waist circumference and sit-and-reach) for participants that have complete data for all five tests. "Fitness-partial" reflects the mean of the fitness z-scores for participants who have complete data on at least three of the five fitness tests.

\section{Covariates}

Covariates included age (years), sex (girls, boys), household income, and body mass index (BMI) based on previous research in older children (Teran-Garcia et al. 2008; Cleland et al. 2009). Furthermore, BMI was chosen as a covariate because international age- and sex-specific reference data can be used to derive BMI z-scores. Household income was parent-reported. The question included six response options ranging from $<\$ 20,000$ to $>\$ 100,000$ of annual income. For BMI, height was measured to the nearest $1.0 \mathrm{~mm}$ using a stadiometer and weight was measured to the nearest $0.1 \mathrm{~kg}$ using a calibrated scale. BMI z-scores were calculated using the World Health Organizations growth standards (de Onis et al. 2007).

\section{Statistical Analysis}

All data analyses were conducted using IBM SPSS Statistics 23.0 software [IBM Corp., Armonk, NY]. To address objective 1, the means and standard deviations were calculated for the raw predicted $\mathrm{VO}_{2}$ max, grip strength, vertical jump, sit-and-reach, and waist circumference values. Frequencies were calculated for the percentage of participants in the "high" and "low" fitness categories for both the push-up and partial curl-up fitness tests according to the Canada Fitness Award "gold standard" (Canada Fitness Award Manual 1986). To address objective 2, linear regression analyses were conducted to examine the association of ST, subjective PA, and objective PA with predicted $\mathrm{VO}_{2}$ max, grip strength, vertical jump, sit-and-reach, waist circumference, fitness-complete, and fitness-partial in individual models. Logistic regressions were employed to examine associations of ST, subjective PA, and objective PA with partial curl- 
ups and push-ups. For all regression models, with the exception of waist circumference, model 1 adjusted for age, sex and household income, and model 2 adjusted for age, sex, household income, and BMI. Due to the high correlation between BMI and waist circumference, BMI was not adjusted for in the models that included waist circumference. To address objective 3 , a moderation analysis was conducted for each association by including a sex $\times$ PA or sex $\times$ ST interaction term in the model. When statistically significant interaction terms were observed, sexstratified analyses were conducted. Statistical significance was set at $\mathrm{p}<0.05$.

\section{Results}

To maximize the data collected, those participants with complete data for each association examined were included in the analysis, resulting in a different total number of participants for each analysis. The amount of missing values was highest in the aerobic fitness test (56\%) and second highest for the objective PA measure (30\%). All other PA, ST, and fitness measures had $<5 \%$ of missing values. The high number of missing values $(n=350)$ in the aerobic fitness test was due to participants not reaching $85 \%$ of their predicted max heart rate, which is necessary for accurate predictions of $\mathrm{VO}_{2}$ max in the CPAFLA protocol (2003). Reasons for not meeting the recommended heart rate ceiling included the children's inability to maintain a set cadence or their choice to stop the test early. For the objective PA measure, the high amount of missing values $(n=193)$ was due to participants not returning the logbook or not wearing the step counter for a total of three days.

Participant information, including descriptive information on fitness is presented in Table 1. The mean age of participants was $7.8 \pm 0.6$ years, and approximately half $(52.4 \%)$ of participants were female. Total mean and standard deviation of PA was $12.6 \pm 7.4$ weekly hours when measured via parent-report, and $8645 \pm 2759$ daily steps when measured with a pedometer. 
Average weekly hours of ST was $13.9 \pm 8.1$. The mean scores for the fitness variables tested in the total sample were: $39.4 \pm 2.3\left(\mathrm{ml} \cdot(\mathrm{kg} \cdot \mathrm{min})^{-1}\right.$ for predicted $\mathrm{VO}_{2} \max , 30.8 \pm 6.1 \mathrm{~kg}$ for grip strength, $20.4 \pm 5.1 \mathrm{~cm}$ for vertical jump, $28.1 \pm 6.6 \mathrm{~cm}$ for sit-and-reach, and $59.8 \pm 6.0 \mathrm{~cm}$ for waist circumference. Only $20.5 \%$ and $7.9 \%$ of participants scored in the high push-ups and partial curl-ups groups, respectively.

Results for the linear regression models are presented in Tables 2 and 3. In the fully adjusted models (model 2), positive associations were observed between subjective PA and overall fitness for both the complete $(\beta=0.009,95 \% \mathrm{CI}: 0.001,0.017)$ and the partial $(\beta=0.006$, 95\%CI: $0.000,0.011)$ fitness scores. Objective PA was also positively associated with the fitness-partial ( $\beta=0.025,95 \% \mathrm{CI}$ : 0.007, 0.042). Negative associations approached significance between ST and the fitness-partial score $(p=0.057 ; \beta=-0.005,95 \% \mathrm{CI}:-0.010,0.000)$. No significant associations were observed between objective PA or ST and the fitness-complete score.

For individual fitness measures in the fully adjusted models, subjective and objective PA were positively associated with vertical jump height $(\beta=0.011,95 \% \mathrm{CI}: 0.000,0.022$ and $\beta=0.043,95 \%$ CI: $0.008,0.078$, respectively). No significant associations were observed for sitand-reach or waist circumference.

Associations between the exposure variables and grip strength and the exposure variables and predicted $\mathrm{VO}_{2}$ max were moderated by sex (results not shown); therefore, linear regressions for both of these fitness measures were stratified by sex (Table 3). In the fully adjusted model, subjective PA was positively associated with predicted $\mathrm{VO}_{2} \max (\beta=0.040,95 \% \mathrm{CI}: 0.018$, $0.063)$ and grip strength $(\beta=0.025,95 \% \mathrm{CI}: 0.011,0.040)$ score in boys only. Similarly, boy's objective PA was positively associated with predicted $\mathrm{VO}_{2} \max (\beta=0.084,95 \% \mathrm{CI}: 0.012,0.157)$. 
A negative association with ST and grip strength $(\beta=-0.016,95 \% \mathrm{CI}:-0.028,-0.004)$ was also observed in boys only. No significant associations were observed for PA or ST with predicted $\mathrm{VO}_{2}$ max or grip strength in girls.

Results for the logistic regression are presented in Table 4. In the fully adjusted model, children with higher objective PA were more likely to be in the high push-up group (OR=1.156 95\%CI: 1.054, 1.267). No significant associations were observed between subjective PA or ST and push-ups or partial curl-ups.

\section{Discussion}

This study examined associations between PA, ST, and various fitness measures in a sample of children aged 6- to 10-years. Both objective and subjective PA measures were positively associated with overall fitness, vertical jump, and predicated $\mathrm{VO}_{2}$ max (boys only). Subjective PA was also positively associated with grip strength (boys only), and objective PA was associated with increased likelihood of scoring "high" on the push-up test. Few associations were observed between ST and fitness, apart from grip strength in boys, where a negative association was observed. It is important to note that the size of effects for all associations were small.

For the most part, the fitness testing protocol in the present study matches that used in a nationally representative sample of Canadian children and youth in 2007-2009 (Tremblay et al. 2010). Compared to the 6- to 10 -year old children in Tremblay et al (2010), the participants in our study had higher grip strength and sit-and-reach scores, and lower predicted $\mathrm{VO}_{2}$ max scores. For example, mean fitness scores for boys and girls in the Tremblay et al paper were: grip strength $(25 \mathrm{~kg}$ and $23 \mathrm{~kg})$, sit-and-reach $(24 \mathrm{~cm}$ and $29 \mathrm{~cm})$, and $\mathrm{VO}_{2} \max \left(56.3 \mathrm{ml} \cdot\left(\mathrm{kg} \cdot \mathrm{min}^{-1}\right)\right.$ and 50.7 $\left.\mathrm{ml} \cdot\left(\mathrm{kg} \cdot \mathrm{min}^{-1}\right) ; 2016\right)$, respectively. In our sample, the mean fitness scores for both boys and girls, girls were: grip strength $(32 \mathrm{~kg}$ and $30 \mathrm{~kg})$, sit-and-reach $(26 \mathrm{~cm}$ and $31 \mathrm{~cm})$, and $\mathrm{VO}_{2} \max (40.53$ 
$\mathrm{ml} \cdot\left(\mathrm{kg} \cdot \mathrm{min}^{-1}\right)$ and $\left.38.6 \mathrm{ml} \cdot\left(\mathrm{kg} \cdot \mathrm{min}^{-1}\right)\right)$, respectively. It is important to note that the national sample only presented predicted $\mathrm{VO}_{2}$ max scores for 8- to10-year olds. Differences in mean fitness cores between samples could also be due to the fact that the Edmonton region sample had slightly lower BMI scores than the national sample and higher median income than reported in the 2006 census data (Statistics Canada 2010). Comparing the findings of the present study to other Canadian and international studies is difficult due to the differences in fitness test procedures.

The association between objective PA and fitness in children 5- to 17-years of age across 38 studies has recently been summarized in a systematic review on PA and health indicators (Poitras et al. 2016). Eighteen out of 29 observational studies, which were all cross-sectional, looked at total PA using both pedometers $(n=4)$ and accelerometers $(n=14)$ and all reported associations between an aerobic fitness measure and total PA (Poitras et al. 2016). However, aerobic fitness findings in the nine included experimental studies were mixed (Poitras et al. 2016). In the present study, a significant association was observed between objective total PA and aerobic fitness in boys only, suggesting that sex may be an important effect modifier to include when examining this relationship in future research. This finding may be because of the maturation differences between boys and girls in relation to their fitness levels or the differences in activity duration between boys and girls in this age group. For example, boy's fitness tends to increase before and after puberty (Beunen and Thomis 2000; Gallahue and Ozman 2006) and boys take more steps in a day compared to girls (Cameron et al. 2016).

Ten observational studies in the Poitras et al. (2016) review examined the association between PA and muscular strength, and the results were mixed. Similarly in the present study, some significant associations were observed between objective PA and vertical jump, push-ups 
and fitness-partial scores but not for grip strength, sit-and-reach, or predicted $\mathrm{VO}_{2}$ max. Of the 38 included studies in the Poitras et al review, only six had a mean age similar to our study $(\leq 8$ years of age) and none of those included measures of muscular strength or muscular endurance. Therefore, including both aerobic and muscular fitness in the present sample of 6- to 10 -year olds provides novel insight into the associations between objectively measured PA and fitness in young children.

Subjective and objective measures of PA can potentially capture different types of movements or activities (i.e., upper body movements or water activities). For example, a child who frequently plays on the monkey bars would record less steps, but would likely record higher subjective PA. This type of activity may correspond to good grip strength, but it may not impact VO2 max. Additionally, it is likely that the subjective measures captured by the parent-proxy in the present study represented time spent in organized activities that could be of higher intensity. Conversely, the objective measures of steps would have captured all activity performed throughout the day, including lower intensity and incidental activities. These differences between measures could potentially explain the different associations observed between objective and subjective PA and fitness. Specifically in this study, subjective PA was positively associated with grip strength (in boys only) and vertical jump, which indicates that the types of activities captured by the PA questionnaire may be positively associated with muscular strength in young children. Upper and lower body strength (push-up test and vertical jump test) were also associated with the objective PA measures. Therefore, both measures of PA appeared important for children's upper and lower body muscular fitness in this sample of children.

In addition to PA, the present study also examined ST as a correlate of fitness in children. Currently, two systematic reviews have looked at the relationship between sedentary behaviour 
and various health indicators, including fitness, in children and youth. A 2011 review (Tremblay et al. 2011) included 15 studies that assessed fitness as a health indicator. More than two hours of ST per day was associated with lower predicted $\mathrm{VO}_{2}$ max, aerobic fitness, or muscular strength in 10 of the 15 studies (Tremblay et al. 2011). However, only four of these studies assessed muscular strength or endurance, and only one study had a mean age younger than 8 years of age (Tremblay et al. 2011). A 5-year update to the Tremblay et al. (2011) review, which included 21 new studies, found consistent findings for total ST and TV viewing with fitness (Carson et al. 2016). However, computer and video game usage alone was not consistently associated with fitness. Of the 15 studies that specifically examined the association between ST and fitness, only two included a sample with a mean age of less than 8 years (Ciesla et al. 2014; Drenowatz et al. 2014). The age differences could potentially explain inconsistent findings between the schoolaged children reviews and the present study. It is possible that the association between ST and fitness in young children may not present as strongly as it does with adolescents. Therefore more time may be needed before the amount of ST in which young children engage in has an effect on health related fitness measures. However, given most of the observational evidence is based on cross-sectional data, future research using a longitudinal design is needed to better understand the relationships between ST and fitness over time, especially with a baseline sample of younger children. Also, one recent study has explored the associations between different types of ST on fitness, concluding that television viewing was negatively associated with all measures of strength in children; whereas, video game use was only negatively associated with one measure of strength (Edelson et al. 2016). Therefore, the type of ST children engage in might be an additional avenue that future research and interventions could explore. 
Since few studies have examined the relationship between PA, ST, and fitness in children under 10 years of age, future research is needed to confirm findings from the present study. For instance, understanding the age at which PA and ST begin to influence children's fitness is crucial for determining the optimal time to intervene to address the downward trend in fitness. One potential reason for why limited fitness data is available in young children is because of the paucity of fitness measures validated for this age group. Consequently, future research that provides standardized measures of physical fitness that are valid and reliable in young children will allow for better comparisons between studies. Furthermore, the majority of children's fitness research has only measured cardiovascular fitness (Poitras et al., 2016). Given the findings from the present study suggest associations may differ depending on the type of fitness measured, future research should also consider other measures of health-related fitness. Lastly, it may be important to consider the distinction between different intensities of PA, in particular moderateintensity PA and vigorous-intensity PA, on fitness using objective measures such as accelerometers. Findings from this research could help inform whether certain intensities of PA should be targeted in future interventions.

The main strengths of this study are the young age of the participants, the relatively large sample size of Canadian children, and the inclusion of muscular strength and endurance measures of fitness. This study is not without limitations. Though the prediction of child $\mathrm{VO}_{2}$ max is consistent with previous research (Tremblay et al. 2010), the equation has not been validated in children; therefore, the aerobic fitness results should be interpreted with caution. Additionally, the psychometric properties of the ST questionnaire are unknown; therefore, information bias may have been present, resulting in ST being underestimated or overestimated. Also, due to the nature of fitness testing, some fitness tests had large amounts of missing data, 
which reduced the sample size, and thus power to detect the effects for some associations. Furthermore, the cross-sectional design prevents us from making causal claims about the findings; therefore, reserve causation cannot be ruled out. Finally, the behaviour measures used in the present study did not assess the entire movement behaviour continuum throughout the children's day, including sedentary time, light-intensity PA and moderate-to-vigorous-intensity PA. It is possible that the intensity of PA may be more strongly associated with fitness than duration, which was not captured by our objective pedometer measure (Blair et al. 2001).

\section{Conclusion}

PA of young children in Edmonton was associated with overall fitness and some individual components of aerobic and muscular fitness. However, in contrast to studies in older children, the associations between ST and fitness measures were primarily null in this age group. Though the association between ST and fitness in this age group requires further investigation, young children may be an optimal age to target PA behaviours to reverse the current declining fitness trends. However, future research using longitudinal study designs is needed to confirm these observations and explore the ST associations further.

\section{Conflict of interests}

The authors have no conflict of interest with this manuscript.

\section{Acknowledgements}

The authors thank the participants, their parents, and the participating Edmonton Health Centres for their cooperation in this research. They also thank and acknowledge the contributions of the large number of staff who supported the SHAPEs baseline study and the SHAPEs of Things to Come longitudinal follow-up study. The study was funded by the Canadian Institutes of Health Research (CIHR; grant \# BEO-85866) and the Heart and Stroke Foundation of Canada (grant \# PG-07-0349). VC is supported by a CIHR New Investigator Salary Award. 


\section{References}

Beunen, G. and Thomis, M. 2000. Muscular strength development in children and adolescents. Pediatr. Exerc. Sci. 12(2):174-97. doi:10.1123/pes.12.2.174.

Blair, S. N., Cheng, Y., and Holder, J. S. 2001. Is physical activity or physical fitness more important in defining health benefits? Med. Sci. Sports Exerc. 33(6):379-399. PMID:11427763.

Cameron, C., Craig, C. L., Bauman, A., and Tudor-Locke, C. 2016. CANPLAY study: Secular trends in steps/day amoungst 5-19 year-old Canadians between 2005 and 2014. Prev. Med. 86:28-33. PMID:26757400.

Canada Fitness Award Manual. 1986. Government of Canada: Fitness and Amateur Sport. Publication No. H93-91

CSEP. 2003. Canadian Physical Activity, Fitness and Lifestyle Approach (CPAFLA), $3^{\text {rd }}$ ed. Canadian Society for Exercise Physiology, Ottawa, Ont. Canada.

CSEP. 2014a. Physical Activity Readiness Questionnaire [online]. Available from http://csep.ca/cmfiles/publications/parq/par-q.pdf [accessed 14 April 2016]

CSEP. 2014b. Canadian sedentary behaviour guidelines for children -5 to 11 years [online]. Canadian Society for Exercise Physiology. Available from http://www.csep.ca/CMFiles/Guidelines/CSEP_SBGuidelines_child_en.pdf [accessed 18 April 2016].

Carson, V., Hunter, S., Kuzik, N., Gray, C.E., Poitras, V.J., Chaput, J., et al. 2016. Systematic review of sedentary behaviour and health indicators in school-aged children and youth: an update. Appl. Physiol. Nutr. Metab. 41(6):s240-s265. doi:10.1139/apnm-2015-0630. 
Chiu, L. L., Wu, Y. F., Tang, M. T., Yu, H. C., Hsieh, L. L. and Hsieh, S.S. 2011. ACTN3 Genotype and Swimming Performance in Taiwan. Int. J. Sports. Med. 32(6):476-480. doi:10.1055/s-0030-1263115.

Cleland, V. J., Ball, K., Magnussen, C., Dwyer, T., and Venn, A. 2009. Socioeconomic position and the tracking of physical activity and cardiorespiratory fitness from childhood to adulthood. Am. J. Epidemiol. 170(9):1069-1077. doi:10.1093/aje/kwp271.

Ciesla, E., Mleczko, E., Bergier, J., Markowska, M., and Nowak-Starz, G. 2014. Health-Related Physical Fitness, BMI, physical activity and time spent at a computer screen in 6 and 7year-old children from rural areas in Poland. Ann. Agric. Environ. Med. 21(3):617-621. PMID:25292140.

Colley, R. C., Garriguet, D., Janssen, I., Craig, C. L., Clarke, J., and Tremblay, M. S. 2011. Physical activity of Canadian children and youth: Accelerometry results from the 20072009 Canadian Health Measures Survey. Statistics Canada, Health Reports. 22(1):15-23.

de Onis, M., Onyango, A. W., Borghi, E., Siyam, A., Nishida, C., and Siekmann, J. 2007. Development of WHO growth reference for school-aged children and adolescents. Bulletin of the World Helath Organization. 85(9):660-667. doi: 10.2471/BLT.07.043497.

Drenowatz, C., Kobel, S., Kettner, S., Kesztyus, D., and Steinacker, J. M. 2014. Interaction of sedentary behaviour, sports participation and fitness with weight status in elementary school children. Eur. J. Sport Sci. 14(1):100-105. PMID:24533500.

Edelson, L. R., Mathias, K. C., Fulgoni, V. L., and Karagounis, L. G. 2016. Screen-based sedentary behaviour and associations with functional strength in 6-15 year-old children in the United States. BMC Public Health. 16:116. doi:10.1186/s12889-016-2791-9. 
Edwards, J., Evans, J., and Brown, A. D. 2008. Using routine growth data to determine overweight and obesity prevalence estimates in preschool children in the Capital health Region of Alberta. Can. J. Public Health. 99:91-94. PMID:18457279.

Froberg, K. 2014. Relation between physical activities, fitness, muscle strengths and health: findings from the European Youth Heart Study (EYHS). Education. Physical Training. Sport. 93(2):10-20.

Gallahue, D. L. and Ozman, J. C. 2006. Understanding motor development: Infants, children, adolescents, adults. 6th ed. Boston: McGrawHill.

Garcia-Artero, E., Ortega, F. B., Ruiz, J. R., Mesa, J. L., Delgado, M., Gonzalez-Gross, M., et al. 2007. Lipid and metabolic profiles in adolescents are affected more by physical fitness than physical activity (ANEVA Study). Rev. Esp. Cardiol. 60(6):581-588.

Government of Alberta. 2015. Health: Annual Report 2014-15.

Grontved, A., Ried-larsen, M., Ekelunf, U., Froberg, K., Brage, S., and Andersen, L. B. 2013. Independent and combined associations of muscle strength and cardiorespiratory fitness in youth with insulin resistance and $\beta$-cell function in young adulthood. Diabetes Care. 36(9):2575-258. doi: 10.2337/dc12-2252.

Janssen, I. and Leblanc, A. G. 2010. Systematic review of the health benefits of physical activity and fitness in school-aged children and youth. Int. J. Behav. Nutr. Phys. Act. 7(40). doi:10.1186/1479-5868-7-40.

Harris, K. C., Kuramoto, L. K., Schulzer, M., and Retallack, J. E. 2009. Effect of school-based physical activity interventions on body mass index in children: a meta-analysis. Can. Med. Assoc. J. 180(7):719-726. doi:10.1503/cmaj.080966. 
Hjorth, M. F. 2014. Fatness predicts decreased physical activity and increased sedentary time, but not vice versa: Support form a longitudinal study in 8- to 11-year old children. Int. J. Obes. (Lond). 38(7):959-965. doi:10.1038/ijo.2013.229.

Kostek, M., Hubal, M., and Pescatello, L. S. 2011. The role of genetic variation in muscle strength. Am. J. Lifestyle Med. 5(2):156-170. doi:10.1177/1559827610387251.

Lammle, L., Woll, A., Mensink, G. B., and Bos, K. 2013. Distal and proximal factors of health behaviours and their associations with the health in children and adolescents. Int. J. Environ. Res. Public Health. 10(7):2944-2978. doi:10.3390/ijerph10072944.

Ling, J. and King, K. M. 2015. Measuring physical activity of elementary school children with unsealed pedometers: Compliance, reliability, and reactivity. J. Nurs. Meas. 23(2):271286. doi:10.1891/1061-3749.23.2.271.

Metcalf, B., Henley, W., and Wilkin, T. 2012. Effectiveness of intervention on physical activity of children: systematic review and meta-analysis of controlled trials with objectively measured outcomes (EarlyBird 54). BMJ. 345:e5888.

Ortega, F. B., Ruiz, J. R., Castillo, M. J., and Sjostrom, M. 2008. Physical fitness in childhood and adolescence: a powerful marker of health. Int. J. Obes. (Lond). 32(1):1-11. doi:10.1038/sj.ijo.0803774.

ParticipACTION. (2016). The ParticipACTION report card on physical activity for children and youth. Available from http://www.participaction.com/en-ca/thought-leadership/report$\operatorname{card} / 2016$

Poitras, V. J., Gray, C. E., Borghese, M. M., Carson, V., Chaput, J., Janssen, I., et al. 2016. Systematic review of the relationships between objectively-measured physical activity 
and health indicators in school-aged children and youth. Appl. Physiol. Nutr. Metab. 41(6):s197-239. doi:10.1139/apnm-2015-0663.

Santos, R., Mota, J., Okely, A. D., Pratt, M., Moreira, C., Coelho-e-Silva, M. J., et al. 2014. The independent associations of sedentary behaviour and physical activity on cardiorespiratory fitness. Br. J. Sports Med. 48(20):1508-1512. doi:10.1136/bjsports2012-091610.

Shields, M. 2006. Overweight and obesity among children and youth. Health Reports (Statistics Canada, 82-003). 17(3):27-42.

Showell, N. N., Fawole, O., Segal, J., Wilson, R. F., Cheskin, L. J., Bleich, S. N., et al. 2013. A systematic review of home-based childhood obesity prevention studies. Pediatrics. 132(1):e193-200. doi:10.1542/peds.2013-0786.

Sirard, J. R. and Pate, R. R. 2001. Physical activity assessment in children and adolescents. Sports Med. 31(6):439-454. PMID:11394563.

Smith, J. J., Eather, N., Morgan, P. J., Plotnikoff, R. C., Faigenbaum, A. D., and Lubans, D. R. 2014. The health benefits of muscular fitness for children and adolescents: a systematic review and meta-analysis. Sports Med. 44(9):1209-1223. doi:10.1007/s40279-014-01964.

Statistics Canada. 2010. Median household income for Canada, provinces and territories, 1996, 2001 and 2006 censuses [online]. Available from http://www12.statcan.ca/censusrecensement/2006/dp-pd/92-596/P2-2.cfm?Lang=eng\&T=PR\&LINE_ID=408 [accessed 14 April 2016]. 
Stearns, J. A., Rhodes, R., Ball, G. D. C., Boule, N., Veugelers, P. J., Cutumisu, N., et al. 2016. A cross-sectional study of the relationship between parents' and children's physical activity. BMC Public Health. 16:1129. Doi:10.1186/s12889-016-3793-3.

Telford, A., Salmon, J., Jolley, D., and Crawford, D. 2004. Reliability and validity of physical activity questionnaires for children: The children's leisure activities survey (CLASS). Pediatr. Exerc. Sci. 16(1):64-78.

Teran-Garcia, M., Rankinen, T., and Bouchard, C. 2008. Genes, exercise, growth, and the sedentary, obese child. J. Appl. Physiol. 105(3):988-1001. doi 10.1152/japplphysiol.00070.2008

Tomkinson, G. R., Leger, L. A., Olds, T. S., and Cazorla, G. 2003. Secular trends in the performance of children and adolescents (1980-2000). Sports Med. 33:85-300. PMID:12688827.

Tremblay, M. S., Shields, M., Laviolette, M., Craig, C. L., Janssen, I., and Gorber, S. C. 2010. Fitness of Canadian children and youth: Results from the 2007-2009 Canadian Health Measures Survey. Health Rep. 21(1). Statistics Canada, Catalogue no. 82-003-XPE.

Tremblay, M. S., LeBlanc, A. G., Kho, M. E., Saunders, T. J., Larouche, R., Colley, R. C., et al. 2011. Systematic review of sedentary behaviour and health indicators in school-aged children and youth. Int. J. Behav. Nutr. Phys. Act. 8(98). doi: 10.1186/1479-5868-8-98.

Van Deutekom, A. W., Chinapaw, M. J., Vrijkotte, T. G., and Gemke, R. J. 2015. The association of birth weight and infant growth with physical fitness at 8-9 years of age-the ABCD study. Int. J. Obes. (Lond). 39(4):593-600. doi:10.1038/ijo.2014.204. 
Weller, I. M., Thomas, S. G., Gledhill, N., Paterson, D., and Quinney, A. 1995. A study to validate the modified Canadian aerobic fitness test. Can. J. Appl. Physiol. 20(2):211-221. PMID:7640647. 
Table 1. Participant information and descriptive statistics of a sample of Edmonton children 6- to 10-years of age.

\begin{tabular}{|c|c|c|c|}
\hline Variables & Girls & Boys & Total \\
\hline \multicolumn{4}{|l|}{ Gender (\%) } \\
\hline Male & - & - & 47.6 \\
\hline \multirow[t]{2}{*}{ Female } & - & - & 52.4 \\
\hline & & & $\mathrm{N}=649$ \\
\hline \multirow[t]{2}{*}{ Age (years) } & $7.8( \pm 0.6)$ & $7.8( \pm 0.6)$ & $7.8( \pm 0.6)$ \\
\hline & & & $\mathrm{N}=649$ \\
\hline \multicolumn{4}{|l|}{ BMI (\%) } \\
\hline Non-overweight & 78.6 & 77.6 & 78.1 \\
\hline \multirow[t]{2}{*}{ Overweight/Obese } & 21.8 & 22.4 & 21.9 \\
\hline & & & $\mathrm{n}=628$ \\
\hline \multicolumn{4}{|l|}{ Household Income (\%) } \\
\hline$<\$ 20,000$ & 1.3 & 2.1 & 1.7 \\
\hline$\$ 20-39,999$ & 1.3 & 2.1 & 1.7 \\
\hline$\$ 40-59,999$ & 7.9 & 5 & 6.5 \\
\hline$\$ 60-79,999$ & 12.7 & 9.6 & 11.2 \\
\hline$\$ 80-99,999$ & 19 & 22.5 & 20.6 \\
\hline \multirow[t]{2}{*}{$>\$ 100,000$} & 57.9 & 58.6 & 58.2 \\
\hline & & & $\mathrm{n}=596$ \\
\hline \multirow[t]{2}{*}{ Physical Activity (hours/week) } & $12.6( \pm 7.6)$ & $12.6( \pm 7.0)$ & $12.6( \pm 7.4)$ \\
\hline & & & $n=631$ \\
\hline \multirow[t]{2}{*}{ Physical Activity (steps/day) } & $8171.7(2641.2)$ & $9185.1(2796.1)$ & $8645.1( \pm 2758.5)$ \\
\hline & & & $\mathrm{n}=456$ \\
\hline \multirow[t]{2}{*}{ Screen Time (hours/week) } & $12.4( \pm 7.2)$ & $15.6( \pm 8.7)$ & $13.9( \pm 8.1)$ \\
\hline & & & $n=630$ \\
\hline \multicolumn{4}{|l|}{ Fitness Scores } \\
\hline $\mathrm{VO}_{2} \max \left(\mathrm{ml} \cdot(\mathrm{kg} \cdot \min )^{-1} ; \mathrm{n}=285\right)$ & $38.6( \pm 2.1)$ & $40.5( \pm 2.1)$ & $39.4( \pm 2.3)$ \\
\hline Grip strength $(\mathrm{kg} ; \mathrm{n}=639)$ & $29.7( \pm 5.6)$ & $32.1( \pm 6.3)$ & $30.8( \pm 6.1)$ \\
\hline Vertical Jump (cm; n=641) & $20.1( \pm 5.0)$ & $20.7( \pm 5.1)$ & $20.4( \pm 5.1)$ \\
\hline Sit-and-Reach $(\mathrm{cm} ; \mathrm{n}=641)$ & $30.7 \pm 6.3)$ & $26.4( \pm 6.2)$ & $28.7( \pm 6.6)$ \\
\hline $\begin{array}{l}\text { Waist Circumference }(\mathrm{cm} \text {; } \\
\mathrm{n}=642)\end{array}$ & $60.0( \pm 6.2)$ & $60.0( \pm 6.2)$ & $59.8( \pm 6.0)$ \\
\hline \multicolumn{4}{|l|}{ Pushups $(\% ; n=639)$} \\
\hline High & 28.7 & 11.5 & 20.5 \\
\hline Low & 71.3 & 88.5 & 79.5 \\
\hline \multicolumn{4}{|l|}{ Partial Curl-ups $(\% ; n=636)$} \\
\hline High & 6.9 & 8.9 & 7.9 \\
\hline Low & 93.1 & 91.1 & 92.1 \\
\hline
\end{tabular}

Note: Data presented as mean ( \pm standard deviation) or as percentage $(\%)$. 
Table 2. Associations of vertical jump, sit-and-reach, waist circumference, and fitness scores (complete and partial) with objective physical activity (steps/day/1000), subjective physical activity (hours/week) and screen time (hours/week) in children 6- to 10-years of age.

\begin{tabular}{|c|c|c|c|c|c|}
\hline & $\begin{array}{l}\text { Vertical jump } \\
\beta(95 \% \mathrm{CI})\end{array}$ & $\begin{array}{l}\text { Sit-and-reach } \\
\qquad \beta(95 \% \mathrm{CI})\end{array}$ & $\begin{array}{c}\text { Waist } \\
\text { circumference } \\
\beta(95 \% \mathrm{CI})\end{array}$ & $\begin{array}{l}\text { Fitness-complete } \\
\qquad \beta(95 \% \mathrm{CI})\end{array}$ & $\begin{array}{l}\text { Fitness-partial } \\
\qquad \beta(95 \% \mathrm{CI})\end{array}$ \\
\hline \multicolumn{6}{|l|}{ Objective $P A$} \\
\hline \multirow[t]{2}{*}{ Model 1} & $0.045(0.010,0.079)^{*}$ & $0.001(-0.034,0.036)$ & $-0.019(-0.054,0.016)$ & $-0.006(-0.035,0.023)$ & $0.014(-0.007,0.034)$ \\
\hline & $\mathrm{p}=0.011$ & $\mathrm{p}=0.951$ & $\mathrm{p}=0.292$ & $\mathrm{p}=0.688$ & $\mathrm{p}=0.194$ \\
\hline \multirow[t]{2}{*}{ Model 2} & $0.043(0.008,0.078)^{*}$ & $0.001(-0.034,0.037)$ & - & $0.005(-0.021,0.030)$ & $0.025(0.007,0.042)^{*}$ \\
\hline & $\mathrm{p}=0.015$ & $\mathrm{p}=0.951$ & & $\mathrm{p}=0.716$ & $\mathrm{p}=0.006$ \\
\hline $\mathrm{n}$ & 455 & 455 & 456 & 200 & 456 \\
\hline \multicolumn{6}{|l|}{ Subjective $P A$} \\
\hline \multirow[t]{2}{*}{ Model 1} & $0.011(0.000,0.022)^{*}$ & $0.000(-0.011,0.011)$ & $-0.001(-0.082,0.060)$ & $0.009(0.000,0.018)^{+}$ & $0.006(0.000,0.012)^{+}$ \\
\hline & $\mathrm{p}=0.049$ & $\mathrm{p}=0.941$ & $\mathrm{p}=0.824$ & $\mathrm{p}=0.058$ & $\mathrm{p}=0.060$ \\
\hline \multirow[t]{2}{*}{ Model 2} & $0.011(0.000,0.022)^{*}$ & $0.000(-0.011,0.011)$ & - & $0.009(0.001,0.017)^{*}$ & $0.006(0.000,0.011)^{*}$ \\
\hline & $\mathrm{p}=0.048$ & $\mathrm{p}=0.941$ & & $\mathrm{p}=0.029$ & $\mathrm{p}=0.033$ \\
\hline $\mathrm{n}$ & 624 & 624 & 625 & 271 & 625 \\
\hline \multicolumn{6}{|l|}{ Subjective $S T$} \\
\hline \multirow[t]{2}{*}{ Model 1} & $-0.004(-0.014,0.006)$ & $-0.005(-0.015,0.005)$ & $0.007(-0.004,0.017)$ & $-0.005(-0.013,0.004)$ & $-0.003(-0.009,0.003)$ \\
\hline & $\mathrm{p}=0.442$ & $\mathrm{p}=0.305$ & $\mathrm{p}=0.214$ & $\mathrm{p}=0.241$ & $\mathrm{p}=0.314$ \\
\hline \multirow[t]{2}{*}{ Model 2} & $-0.003(-0.014,0.007)$ & $-0.005(-0.015,0.005)$ & - & $-0.007(-0.014,0.001)$ & $-0.005(-0.010,0.000)^{+}$ \\
\hline & $\mathrm{p}=0.577$ & $\mathrm{p}=0.304$ & & $\mathrm{p}=0.064$ & $\mathrm{p}=0.057$ \\
\hline $\mathrm{n}$ & 641 & 641 & 642 & 283 & 642 \\
\hline
\end{tabular}

Note: Fitness-complete includes participants with complete data for vertical jump, sit-and-reach, $\mathrm{VO}_{2}$ max, grip strength, and waist circumference. Fitness-partial includes participants with complete data for at least three components of fitnesscomplete. Model 1 is adjusted for age, sex, and household income. Model 2 is adjusted for age, sex, household income, and BMI.

${ }^{*} \mathrm{p}<0.05 ;{ }^{+} \mathrm{p}<0.07$ 
Table 3. Sex specific associations of objective (steps/day/1000) and subjective (hours/week) physical activity and screen time (hours/week) with grip strength and predicted $\mathrm{VO}_{2}$ max scores in children 6- to 10-years of age.

\begin{tabular}{|c|c|c|c|c|}
\hline & $\begin{array}{c}\mathrm{VO}_{2} \max (\text { boys }) \\
\beta(95 \% \mathrm{CI})\end{array}$ & $\begin{array}{c}\text { Grip strength (boys) } \\
\beta(95 \% \mathrm{CI})\end{array}$ & $\begin{array}{c}\mathrm{VO}_{2} \max (\text { girls }) \\
\beta(95 \% \mathrm{CI})\end{array}$ & $\begin{array}{c}\text { Grip strength (girls) } \\
\beta(95 \% \mathrm{CI})\end{array}$ \\
\hline \multicolumn{5}{|l|}{ Objective PA } \\
\hline \multirow[t]{2}{*}{ Model 1} & $0.065(-0.016,0.147)$ & $0.022(-0.028,0.072)$ & $0.015(-0.053,0.083)$ & $0.023(-0.025,0.071)$ \\
\hline & $\mathrm{p}=0.116$ & $\mathrm{p}=0.395$ & $\mathrm{p}=0.658$ & $\mathrm{p}=0.343$ \\
\hline \multirow[t]{2}{*}{ Model 2} & $0.084(0.012,0.157)^{*}$ & $0.039(-0.006,0.084)$ & $0.028(-0.036,0.092)$ & $0.035(-0.010,0.080)$ \\
\hline & $\mathrm{p}=0.024$ & $\mathrm{p}=0.086$ & $\mathrm{p}=0.394$ & $\mathrm{p}=0.131$ \\
\hline $\mathrm{n}$ & 78 & 221 & 124 & 242 \\
\hline \multicolumn{5}{|l|}{ Subjective PA } \\
\hline \multirow[t]{2}{*}{ Model 1} & $0.040(0.015,0.066)^{*}$ & $0.025(0.009,0.041)^{*}$ & $0.010(-0.010,0.031)$ & $0.003(-0.011,0.017)$ \\
\hline & $\mathrm{p}=0.002$ & $\mathrm{p}=0.002$ & $\mathrm{p}=0.326$ & $p=0.664$ \\
\hline \multirow[t]{2}{*}{ Model 2} & $0.040(0.018,0.063)^{*}$ & $0.025(0.011,0.040)^{*}$ & $0.010(-0.009,0.030)$ & $0.003(-0.010,0.016)$ \\
\hline & $\mathrm{p}=0.001$ & $\mathrm{p}=0.001$ & $\mathrm{p}=0.306$ & $\mathrm{p}=0.668$ \\
\hline $\mathrm{n}$ & 115 & 294 & 158 & 328 \\
\hline \multicolumn{5}{|l|}{ Subjective ST } \\
\hline \multirow[t]{2}{*}{ Model 1} & $0.002(-0.020,0.024)$ & $-0.016(-0.029,-0.002)^{*}$ & $-0.002(-0.025,0.021)$ & $0.000(-0.015,0.016)$ \\
\hline & $\mathrm{p}=0.848$ & $\mathrm{p}=0.018$ & $\mathrm{p}=0.903$ & $\mathrm{p}=0.932$ \\
\hline \multirow[t]{2}{*}{ Model 2} & $0.001(-0.018,0.021)$ & $-0.016(-0.028,-0.004)^{*}$ & $-0.007(-0.029,0.014)$ & $-0.004(-0.019,0.010)$ \\
\hline & $\mathrm{p}=0.879$ & $\mathrm{p}=0.006$ & $\mathrm{p}=0.540$ & $\mathrm{p}=0.451$ \\
\hline $\mathrm{n}$ & 120 & 303 & 165 & 336 \\
\hline
\end{tabular}

Note: Model 1 is adjusted for age and household income. Model 2 is adjusted for age, household income, and BMI.

$* \mathrm{p}<0.05$ 
Table 4. Objective (steps/day/1000) and subjective (hours/week) physical activity, screen time (hours/week) and associations with a high push-up or partial curl-up score (gold-standard score with the Canada Fitness Award) in children 6- to 10 -years of age.

\begin{tabular}{ccc}
\hline & Push-up & Partial curl-up \\
OR $(95 \% \mathrm{CI})$ & OR $(95 \% \mathrm{CI})$ \\
\hline Objective PA & & \\
Model 1 & $1.165(1.064,10227)^{*}$ & $0.863(0.735,1.012)$ \\
Model 2 & $\mathrm{p}=0.001$ & $\mathrm{p}=0.070$ \\
$\mathrm{n}$ & $1.156(1.054,1.267)^{*}$ & $0.860(0.735,1.010)^{+}$ \\
Subjective PA & $\mathrm{p}=0.002$ & $\mathrm{p}=0.066$ \\
Model 1 & 420 & 420 \\
& & $1.029(0.990,1.070)$ \\
Model 2 & $1.017(0.989,1.045)$ & $\mathrm{p}=0.152$ \\
$\mathrm{n}$ & $\mathrm{p}=0.235$ & $1.029(0.988,1.070)$ \\
Subjective ST & $1.019(0.992,1.048)$ & $\mathrm{p}=0.158$ \\
Model 1 & $\mathrm{p}=0.175$ & 575 \\
& 578 & $1.020(0.984,1.058)$ \\
Model 2 & & $\mathrm{p}=0.270$ \\
$\mathrm{n}$ & $1.006(0.979,1.033)$ & $1.020(0.984,1.058)$ \\
& $\mathrm{p}=0.680$ & $\mathrm{p}=0.255$ \\
& $1.006(0.979,1.034)$ & 590 \\
\hline
\end{tabular}

Note: Model 1 is adjusted for age, sex and household income. Model 2 is adjusted for age, sex, household income, and BMI.

$* \mathrm{p}<0.05$

$+\mathrm{p}<0.07$ 
